\title{
Constant Envelope Precoding with Extended Degrees of Freedom through Per-user Symbol Scaling
}

\author{
Yi Wang, Ning Chen, Fan Liu, Member, IEEE, Ang Li, Member, IEEE, Jianming Zhou, Christos Masouros, \\ Senior Member, IEEE
}

\begin{abstract}
In this letter, we propose a novel constant envelope precoding (CEP) design for multi-user multi-input single-output (MU-MISO) downlink, where we exploit the extra degrees of freedom (DoFs) by allowing individual rotation and scaling for each constellation point. By jointly optimizing the transmitting signal matrix and the complex scaling factors, the signal-tointerference-and-noise-ratio (SINR) at the receiver is maximized. The formulated optimization problem is divided into two subproblems and solved by the alternating optimization approach. We further propose a simplified gradient descent algorithm and extend our algorithm to frame-level precoding design. Simulation results show that the proposed algorithms outperform the existing CEP algorithms in terms of both symbol error rate (SER) and computational complexity.
\end{abstract}

Index Terms-Alternating optimization, Constant Envelope precoding, DinkelBach method, Gradient projection, Per-user scaling and rotating.

\section{INTRODUCTION}

A $\mathrm{S}$ a promising technology to reduce the cost and power consumptions in massive MIMO base stations, the constant envelope precoding (CEP) design, which was firstly proposed in [1] by imposing CE constraints on the transmitted signals, has recently gained significant research attentions. Given their favourable peak-to-average power ratio (PAPR) properties, such $\mathrm{CE}$ signals can be transmitted through a low-cost, high-efficiency nonlinear RF power amplifier (PA) without causing any distortion.

It is noteworthy that the non-convex nature of CE constraints poses challenges to the precoding design. By employing the multi-user interference (MUI) as the cost function, the CEP design can be formulated as a non-convex nonlinear leastsquares (NLS) problem, where the local minimum is obtain-

Manuscript received August 24, 2020; revised September 25, 2020 and December 2, 2020; accepted December 28, 2020. This research was supported by the National Nature Science Foundation of China with Grant No.61771047. The associate editor coordinating the review of this letter and approving it for publication was Dr. Marco Gomes. (Corresponding author: Jianming Zhou.)

Y. Wang, N. Chen and J.-M. Zhou are with the School of Information and Electronics, Beijing Institute of Technology, Beijing 100081, China (e-mail: 3120180707, chenning, zhoujm@bit.edu.cn).

F. Liu is with the Department of Electrical and Electronic Engineering, Southern University of Science and Technology, Shenzhen 518055, China(email: liuf6@sustech.edu.cn).

A. Li is with the School of Information and Communications Engineering, Faculty of Electronic and Information Engineering, Xi' an Jiaotong University, Xi'an, Shaanxi 710049, China. (e-mail: ang.li.2020@xjtu.edu.cn).

C. Masouros is with the Department of Electronic and Electrical Engineering, University College London, Torrington Place, London, WC1E7JE, UK. (e-mail: c.masouros@ucl.ac.uk). able by using the gradient descent (GD) algorithm [1]. Due to the CE limitation, however, the symbol error saturation can not be well-addressed in the high SNR regime. As further steps, the cross entropy method (CEO) was proposed in [2] to facilitate the search of the optimum of the CEP design. The work of [3] aimed at CEP design for single-user large-scale MISO channels, which provides a complete characterization of the noise-free receive signal region, under two novel CEP scenarios, wherein the system has the flexibility to either select a subset of antennas, or allocate powers unequally across antennas. More relevant to this work, [4] studied the design of adaptive receiver constellation for CEP in a single user MISO flat-fading channel with arbitrary number of transmit antennas. By relying on the semidefinite relaxation (SDR) method, a novel CEP design was proposed by the same authors in [5] to minimize the maximum SER among all users, where the CE precoding at the transmitter and the constellation scaling and rotation at each user are jointly optimized. Another study by [6] focused on multi-stream transmission, where the transmit antenna grouping and receive beamforming vectors are jointly designed to minimize the maximum SER over all data streams. Nevertheless, they are both computationally expensive in general. By noting the fact that the MUI is known to the $\mathrm{BS}$, the concept of constructive interference (CI) was considered in [7], which reduces the resulting SER of CEP by utilizing constructive MUI resources instead of fully eliminating them. More recently, an efficient Riemannian conjugate gradient (RCG) algorithm was proposed in [8] to solve the CI based CEP problem, which achieves a favorable tradeoff between performance and complexity.

Inspired by the above methods, in our work, we assign a complex coefficient to each constellation point, that hereafter will be referred to as rotating-and-scaling (RS) factor. Differing from [5], in which the scaling factor was optimized as a scalar, we take the independent constellation RS factor for each user and the transmitted signals as optimization variables, with the sum of the receiver's SINR being the objective function. Based on the gradient projection and Dinkelbach methods, a simple alternating optimization (AO) framework is proposed for symbol-level CEP design. It is further extended to a frame-level design, where symbols transmitted within the channel duration share a common RS factor, for the practical purpose of reducing the signalling overhead. Simulation results show that the proposed methods are superior to the existing benchmark techniques in terms of the SER performance. 


\section{SYSTEM MODEL}

We consider an MU-MISO downlink scenario where the base station (BS) equipped with $N$ antennas serves $M$ single-antenna users. The vector of channel gains from the BS antennas to the $k$-th user is denoted by $\mathbf{h}_{k}=$ $\left[h_{1, k}, h_{2, k}, \ldots, h_{N, k}\right]^{T}, k=1, \ldots, M$. Without loss of generality, the channel is assumed to be Rayleigh fading, i.e., $h_{i, k}, i=1, \ldots, N$ subject to i.i.d. standard complex Gaussian distribution. We assume that the BS knows the channel state information (CSI) perfectly, and the imperfect CSI case is designated as our future work. Let $x_{i}$ be the symbol transmitted from the $i$-th antenna of the BS and $\mathbf{x}=\left[x_{1}, x_{2}, \ldots, x_{N}\right]^{T}$ the vector of transmitted symbols. Further, let $\theta_{i}$ be the phase of $x_{i}$ and $P_{T}$ denote the total power of $\mathbf{x}$. The per-antenna CE signal is expressed by [1]:

$$
x_{i}=\sqrt{P_{T} / N} e^{j \theta_{i}}, i=1,2, \ldots, N .
$$

The signal received by the $k$-th user is given by:

$$
y_{k}=\sqrt{P_{T} / N} \sum_{i=1}^{N} h_{i, k} e^{j \theta_{i}}+\omega_{k}, k=1,2, \ldots, M
$$

where $\omega_{k} \sim \mathcal{C N}\left(0, \sigma^{2}\right)$ is additive white Gaussian noise (AWGN) with zero mean and variance $\sigma^{2}$ at the $k$-th receiver.

Let $\mathbf{s}=\left[s_{1}, \ldots, s_{M}\right]^{T} \in \mathbb{C}^{M \times 1}$ be the vector of standard PSK or QAM constellation symbols. To exploit extra degrees of freedom, we set $\boldsymbol{\beta}=\left[\beta_{1}, \ldots, \beta_{M}\right]^{T} \in \mathbb{C}^{M \times 1}$ as the complex vector of RS factors for the constellation symbol of each user. Being multiplied by a complex vector means that each constellation point is allowed to rotate and scale individually. As such, the received signal at the $k$-th user can be expressed as

$$
y_{k}=\sqrt{P_{T}} \beta_{k} s_{k}+\underbrace{\sqrt{P_{T}}\left(\frac{\sum_{i=1}^{N} h_{i, k} e^{j \theta_{i}}}{\sqrt{N}}-\beta_{k} s_{k}\right)}_{\text {MUI }}+\omega_{k}
$$

where $k=1,2, \ldots, M$. The second term stands for the multiuser interference. The received SINR at the $k$-th user is given by

$$
\gamma_{k}=\frac{P_{T}\left|\beta_{k} s_{k}\right|^{2}}{\left|\mathbf{h}_{k}^{T} \mathbf{x}-\sqrt{P_{T}} \beta_{k} s_{k}\right|^{2}+\sigma^{2}} .
$$

\section{Problem Formulation And Decomposition}

Our goal is to maximize users' SINR by jointly designing the transmitted signal $\mathrm{x}$ and RS factors $\boldsymbol{\beta}$, which is equivalent to minimizing the inverse of SINR $r_{k}=1 / \gamma_{k}$. While each user has an independent RS factor, which can be designed to minimize its own $r_{k}$, the signal vector $\mathbf{x}$ is transmitted to all users. As a consequence, it is unlikely for all users to have the maximum SINR at the same time. The cost function is thus set as the sum of the inverse SINR of each user, i.e., $\sum_{k=1}^{M} r_{k}$. Therefore, the CEP design can be formulated into the following optimization problem

$$
\begin{aligned}
& \min _{\mathbf{x}, \beta_{k}} \sum_{k=1}^{M} \frac{\left|\mathbf{h}_{k}^{T} \mathbf{x} / \sqrt{P_{T}}-\beta_{k} s_{k}\right|^{2}+\sigma^{2} / P_{T}}{\left|\beta_{k} s_{k}\right|^{2}} \\
& \text { s.t. }\left|x_{i}\right|^{2}=\sqrt{P_{T} / N}, i=1,2, \ldots, N .
\end{aligned}
$$

It is noteworthy that the optimization variables $\boldsymbol{\beta}$ and $\mathrm{x}$ are independent with each other, in which case the optimization problem can be recast as two subproblems. In what follows, we elaborate on the subproblems for $\mathbf{x}$ and $\boldsymbol{\beta}$, respectively.

\section{A. Subproblem for $\mathbf{x}$}

Given a fixed $\boldsymbol{\beta}$, the objective function can be equivalently expressed as

$$
\min _{\mathbf{x}} \sum_{k=1}^{M}\left|\frac{\mathbf{h}_{k}^{T} \mathbf{x}}{\sqrt{P_{T}} \beta_{k} s_{k}}-1\right|^{2}+\frac{\sigma^{2}}{P_{T}\left|\beta_{k} s_{k}\right|^{2}} .
$$

The second term can be dropped without affecting the result, as $\mathbf{x}$ is the only variable to be considered. By denoting $\mathbf{c}_{k}^{T}=\frac{\mathbf{h}_{k}^{T}}{\sqrt{P_{T} \beta_{k} s_{k}}}$, and $\mathbf{C}=\left[\mathbf{c}_{1}, \mathbf{c}_{2}, \ldots, \mathbf{c}_{M}\right]^{T}$, the first term can be written as the square of the vector's Euclidean norm. Accordingly, (6) can be recast in a compact form as

$$
\begin{aligned}
& \min _{\mathbf{x}}\|\mathbf{C x}-\mathbf{1}\|_{2}^{2} \\
& \text { s.t. }\left|x_{i}\right|^{2}=\sqrt{P_{T} / N}, i=1,2, \ldots, N
\end{aligned}
$$

where 1 is the $M$-dimensional all-one vector. While (7) is a non-convex NLS problem, which has multiple local minima, a near-optimal solution can be obtained by using the gradient projection method, since most of the local minima have been proved to yield small objective values [1]. The gradient of $\mathbf{x}$ in the $t$-th iteration is calculated as

$$
\nabla_{\mathbf{x}^{(t)}} f=2 \mathbf{C}^{H}\left(\mathbf{C x}^{(t)}-\mathbf{1}\right)
$$

where the superscript $H$ denotes the conjugate transpose. We choose the stepsize as $\alpha=1 / \lambda_{\max }$, where $\lambda_{\max }$ is the maximum eigenvalue of $2 \mathbf{C}^{H} \mathbf{C}$, i.e. the Lipschitz constant. The iteration scheme of our gradient projection algorithm can be expressed as

$$
\begin{gathered}
\mathbf{x}^{(t+1)}=\mathbf{x}^{(t)}-\alpha \nabla_{\mathbf{x}^{(t)}} f \\
x_{i}^{(t+1)}=\left(\sqrt{P_{T} / N}\right) \frac{x_{i}^{(t+1)}}{\left|x_{i}^{(t+1)}\right|}, i=1, \ldots, N .
\end{gathered}
$$

Repeat (8)-(10) until $\mathbf{x}$ converges, i.e., $\left\|\mathbf{x}^{(t+1)}-\mathbf{x}^{(t)}\right\|<\Delta$, where $\Delta$ is the maximum tolerance. ${ }^{1}$

\section{B. Subproblem for $\boldsymbol{\beta}$}

In the case that $\boldsymbol{\beta}$ is considered as the optimization variable given a fixed $\mathbf{x}$, the optimization problem can be transformed from $\min \sum_{k=1}^{M} r_{k}$ to $\sum_{k=1}^{M} \min r_{k}$, since all the $\beta_{k}$ and the resulting $r_{k}$ for each user are independent to each other. Thus, there are $k$ unconstrained fractional optimization problems that need to be solved. Let us write the objective function in a more compact form as follows:

$$
\begin{aligned}
\min _{\beta_{k}} r_{k} & =\min _{\beta_{k}} \frac{\left|\mathbf{h}_{k}^{T} \mathbf{x} / \sqrt{P_{T}}-\beta_{k} s_{k}\right|^{2}+\sigma^{2} / P_{T}}{\left|\beta_{k} s_{k}\right|^{2}} \\
& =\min _{\beta_{k}} \frac{g_{1}\left(\beta_{k}\right)}{g_{2}\left(\beta_{k}\right)} .
\end{aligned}
$$

\footnotetext{
${ }^{1}$ We remark here that the convergence results of the gradient projection method for unit-modulus LS problem have already been provided in the literature [9], and will not be repeated here due to the limited space.
} 
The Dinkelbach method can be applied here [10]. The idea behind this method is to relax the fractional objective function into its subtractive counterpart, which is convex. We denote $\beta_{k}^{*}$ and $r_{k}^{*}$ as the optimal solution and the objective function of (11), respectively. As such, the objective function can be recast as

$$
\begin{aligned}
& \min _{\beta_{k}} g_{1}\left(\beta_{k}\right)-r_{k} g_{2}\left(\beta_{k}\right) \\
= & g_{1}\left(\beta_{k}^{*}\right)-r_{k} g_{2}\left(\beta_{k}^{*}\right) .
\end{aligned}
$$

Since $g_{1}\left(\beta_{k}\right)-r_{k} g_{2}\left(\beta_{k}\right)=0$, it is evidently clear that $g_{1}\left(\beta_{k}^{*}\right)-$ $r_{k} g_{2}\left(\beta_{k}^{*}\right) \leq 0$, which means that we have $r_{k}^{*}=\frac{g_{1}\left(\beta_{k}^{*}\right)}{g_{2}\left(\beta_{k}^{*}\right)} \leq$ $r_{k}$. Thus, the optimal solution of (11) can be obtained by iteratively solving (12). By taking a closer look at (12), we note that it is a 1-dimensional quadratic optimization problem. In particular, if $r_{k}<1$, (12) is convex and has the following closed-form solution

$$
\beta_{k}=\frac{\mathbf{h}_{k}^{T} \mathbf{x}}{\sqrt{P_{T}} s_{k}\left(1-r_{k}\right)} .
$$

In the case of $r_{k}>1$, the problem is concave, where there is no analytic solution for the minimization problem. We therefore consider to move towards the descent direction with respect to $\beta_{k}$ by again exploiting the gradient descent technique. We take the negative gradient as the descent direction, with a stepsize $\alpha_{\beta}$. The process is expressed as

$$
\begin{gathered}
\nabla_{\boldsymbol{\beta}_{k}} \boldsymbol{f}=-\frac{s_{k}{ }^{*} \mathbf{h}_{k}^{T} \mathbf{x}}{\sqrt{P_{T}}}+\left(1-r_{k}\right)\left|s_{k}\right|^{2} \beta_{k} \\
\beta_{k}^{\prime}=\beta_{k}-\alpha_{\beta} \nabla_{\boldsymbol{\beta}_{k}} \boldsymbol{f}
\end{gathered}
$$

where $\alpha_{\beta}$ can take the appropriate constant based on the value of the derivative. As per our numerical simulations, it is worth highlighting that by using (14) and (15), $r_{k}$ reaches a value smaller than 1 within a small number of iterations, after which (12) will have an analytic solution. For clarity, we summarize the above procedure for solving (12) as Algorithm 1.

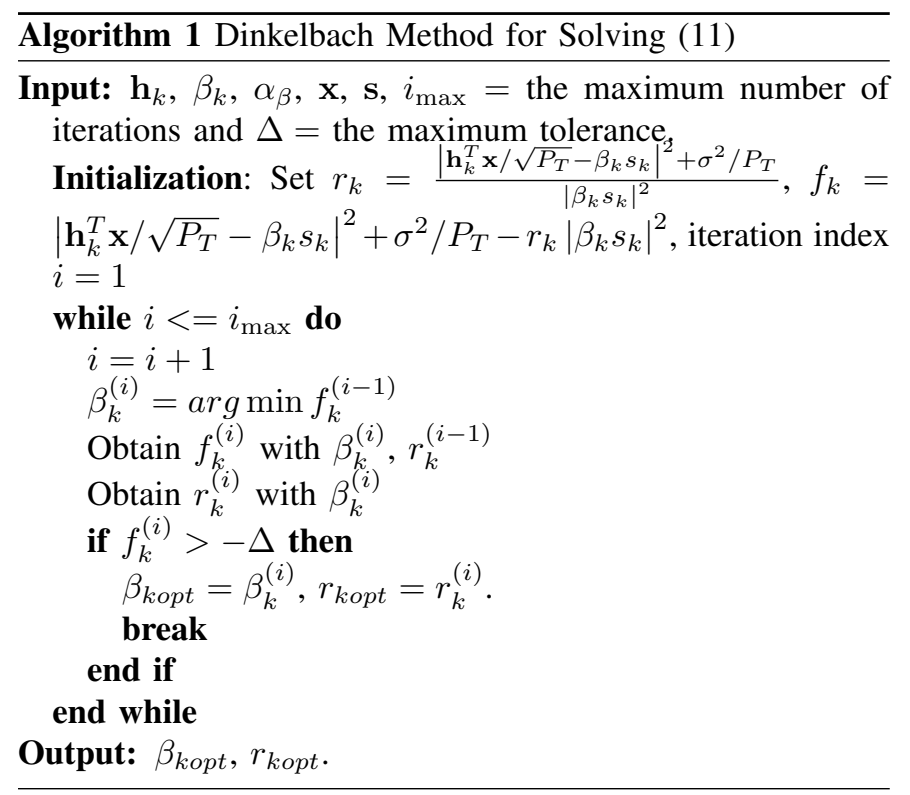

\section{Proposed Algorithm}

\section{A. Conventional Alternating Optimization}

The optimization problem in (5) can be solved via the approach of alternating optimization (AO) [11]. Let us firstly keep the RS vector $\boldsymbol{\beta}$ unchanged, and solve the subproblem (7) to update $\mathbf{x}$. Given a fixed $\mathbf{x}$, we solve the subproblem (11) and update $\boldsymbol{\beta}$. The two subproblems are iteratively solved until the objective function converges. It is worth noting that $r_{k}$ is invovled in this process and generates a temporary value, which would not affect the final convergence. This explains why indexing for $r_{k}$ variable in $\mathrm{AO}$ is different from the Dinkelbach method. We show the specific algorithm in Algorithm 2.

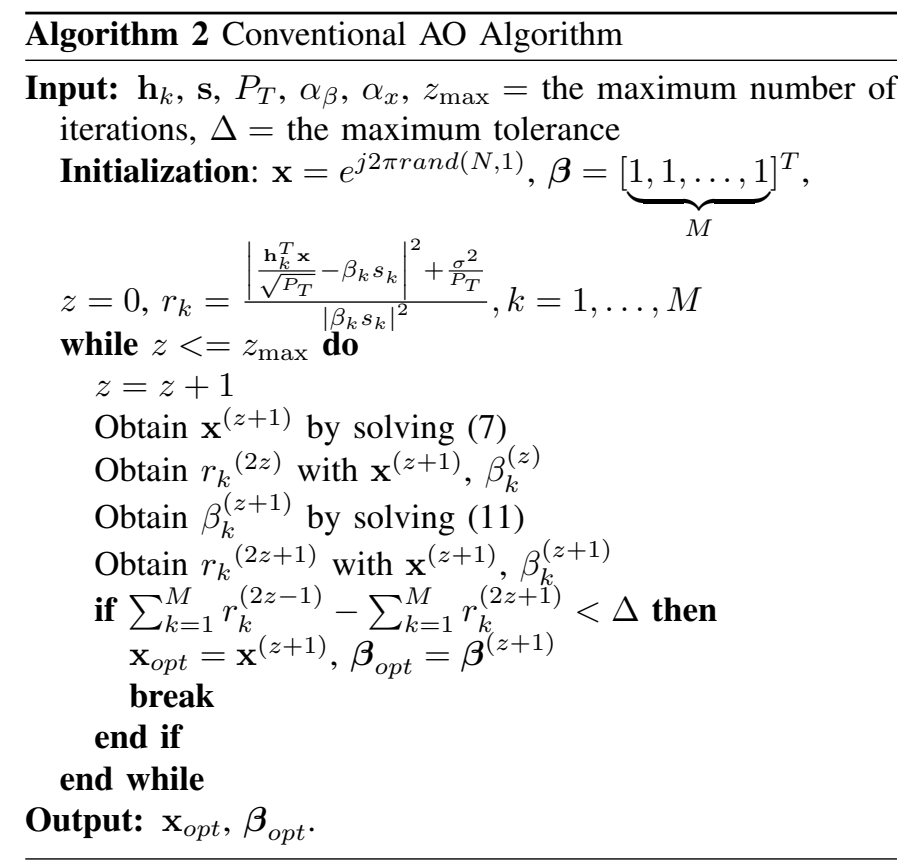

The complexity of this conventional AO algorithm is dominated by the computation of the stepsize for $\mathbf{x}$, where $2 N^{2} M$ flops are needed, leading to a total complexity of $\mathcal{O}\left(N^{2} M\right)$ for each iteration.

\section{B. A Simplified AO Scheme}

To further reduce the computational overhead incurred in the AO algorithm, we propose to simplify the iteration procedure by noting the following facts:

- The gradient projection method with $\mathbf{x}$ as the optimization variable is an algorithm in which the value of objective function strictly declines at every step.

- As long as $f_{k}$ is reset to 0, (16) holds true, which means $\sum_{k=1}^{M} r_{k}^{\prime}<=\sum_{k=1}^{M} r_{k}$. Therefore, the Dinkelbach method with $\boldsymbol{\beta}$ as the optimization variable can ensure decline of objective function values at each step.

$$
f_{k}^{\prime}=\min _{\beta_{k}} f_{k} \leq 0
$$

- Since the algorithms used in both sub-problems can strictly reduce the objective function at each step, the AO can be operated per iteration [12], i.e., in each iteration 
we firstly step toward the descent direction of $\mathbf{x}$ and then of $\boldsymbol{\beta}$, which can also strictly ensure the decline of the objective function.

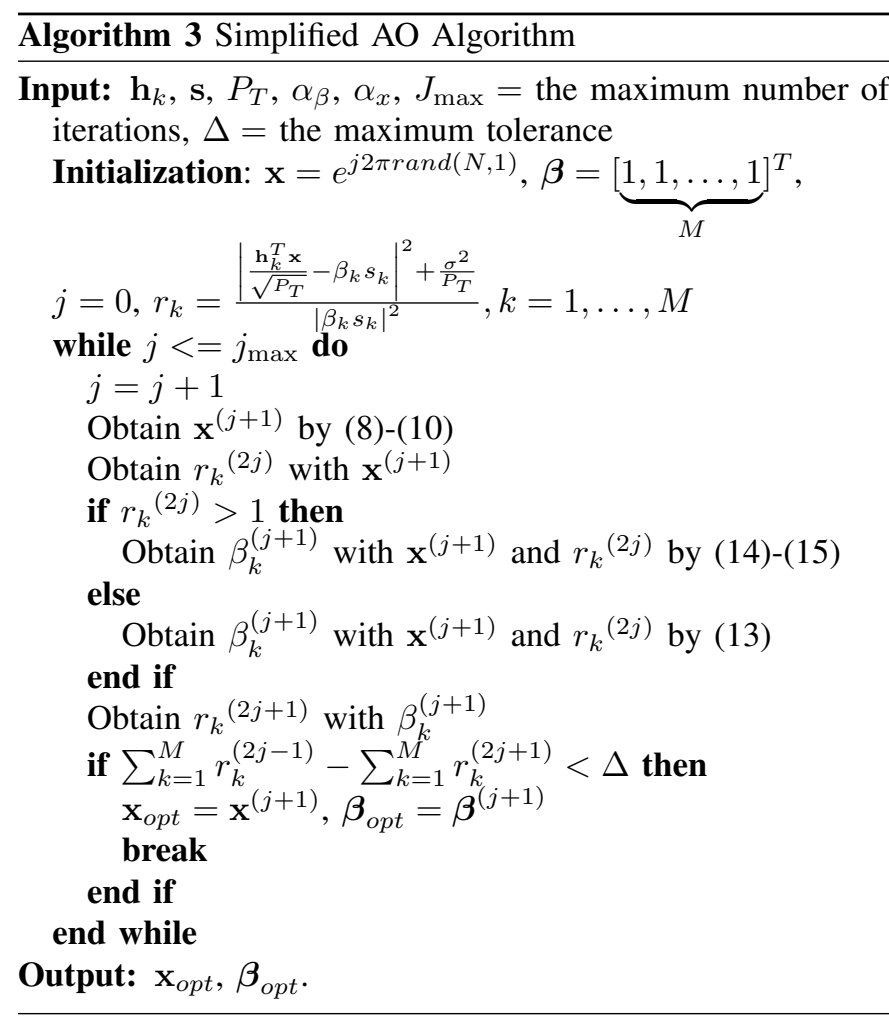

The complexity of the simplified AO algorithm is also $\mathcal{O}\left(N^{2} M\right)$ per iteration, mainly resulting from the computation of the stepsize for $\mathbf{x}$. For the conventional AO algorithm, both subproblems are required to iterate to convergence. Nevertheless, with the same convergence precision, the number of iterations required by the simplified $\mathrm{AO}$ algorithm is much less than that of its conventional counterpart. For the sake of clarity, we show in Algorithm 3 the simplified algorithm, and compare the running time of both algorithms in Fig.1(b).

\section{Frame-level Design}

The above approach is executed on a symbol-by-symbol basis, which relies on the assumption that users need to know the corresponding RS factors for each symbol received. This means that the coefficients $\boldsymbol{\beta}$ should be communicated to the users before transmitting the useful information, which would generate significant signaling overhead. To this end, in this section we extend the proposed symbol-level algorithm to the frame level, i.e., the symbols received by a user in a block fading channel share the same RS factor. Let us assume that the channel keeps unchanged within a transmission block of $L$ symbols. Let $\mathbf{X}$ be a $N \times L$ transmitted signal matrix, with $\mathbf{x}_{l}$ being the symbols transmitted by all the $N$ antennas in the 1-th symbol duration. The vector $\mathbf{s}_{k}$ denotes the $k$-th user's constellation symbols, with $s_{l, k}$ being its $l$-th entry. The received SINR of each user becomes the cumulative signal power divided by the sum of cumulative MUI power and noise power of $L$ symbols. Hence, the optimization problem can be rewritten as

$$
\begin{aligned}
& \min _{\mathbf{X}, \beta_{k}} \sum_{k=1}^{M} \frac{\left\|\mathbf{h}_{k}^{T} \mathbf{X} / \sqrt{P_{T}}-\beta_{k} \mathbf{s}_{k}^{T}\right\|^{2}+\sigma^{2} L / P_{T}}{\left\|\beta_{k} \mathbf{s}_{k}^{T}\right\|^{2}} \\
& \text { s.t. }\left|x_{i, l}\right|^{2}=\sqrt{\frac{P_{T}}{N}}, i=1,2, \ldots, N ; l=1,2, \ldots, L .
\end{aligned}
$$

Further, (17) can be expressed as:

$$
\begin{aligned}
& \min _{\mathbf{x}_{l}, \beta_{k}} \sum_{k=1}^{M} \sum_{l=1}^{L} \frac{\left|\mathbf{h}_{k}^{T} \mathbf{x}_{l} / \sqrt{P_{T}}-\beta_{k} s_{l, k}\right|^{2}+\sigma^{2} / P_{T}}{\left\|\beta_{k} \mathbf{s}_{k}^{T}\right\|^{2}} \\
= & \min _{\mathbf{x}_{l}, \beta_{k}} \sum_{l=1}^{L} \sum_{k=1}^{M}\left|\frac{\mathbf{h}_{k}^{T} \mathbf{x}_{l}}{\sqrt{P_{T}}\left\|\beta_{k} \mathbf{s}_{k}^{T}\right\|}-\frac{\beta_{k} s_{l, k}}{\left\|\beta_{k} \mathbf{s}_{k}^{T}\right\|}\right|^{2}+\frac{\sigma^{2} / P_{T}}{\left\|\beta_{k} \mathbf{s}_{k}^{T}\right\|^{2}} .
\end{aligned}
$$

Let $v_{k}=\left\|\beta_{k} \mathbf{s}_{k}^{T}\right\|, \mathbf{c}_{k}^{T}=\frac{\mathbf{h}_{k}^{T}}{\sqrt{P_{T}} v_{k}}, d_{k}=\frac{\beta_{k} s_{l, k}}{v_{k}}, k=$ $1,2, \ldots, M$. Given a fixed $\boldsymbol{\beta}$ and solve for $\mathbf{X}$, the objective function is

$$
\min _{\mathbf{x}_{l}} \sum_{l=1}^{L}\left\|\mathbf{C} \mathbf{x}_{l}-\mathbf{d}\right\|^{2}
$$

where $\mathbf{C}=\left[\mathbf{c}_{1}, \mathbf{c}_{2}, \ldots, \mathbf{c}_{M}\right]^{T}, \mathbf{d}=\left[d_{1}, d_{2}, \ldots, d_{M}\right]^{T}$. The gradient of $\mathbf{x}_{l}$ in the $\mathrm{t}$-th iretation is calculated as

$$
\nabla_{\mathbf{x}_{l}^{(t)}} f=2 \mathbf{C}^{H}\left(\mathbf{C} \mathbf{x}_{l}^{(t)}-\mathbf{d}\right) .
$$

The stepsize $\alpha$ is the reciprocal of $\lambda_{\max }$, which is the maximum eigenvalue of $2 \mathbf{C}^{H} \mathbf{C}$. Similarly, when we fix $\mathbf{X}$ and solve for $\boldsymbol{\beta}$, the formula is the same as symbol-level algorithm, except that $\mathbf{X}$ and $s_{k}$ are replaced by $\mathbf{X}$ and $\mathbf{s}_{k}^{T}$. This is

$$
\beta_{k}=\frac{\left(\mathbf{h}_{k}^{T} \mathbf{X}\right) \mathbf{s}_{k}^{*}}{\sqrt{P_{T}}\left\|\mathbf{s}_{k}\right\|^{2}\left(1-r_{k}\right)} .
$$

The complexity of frame-level algorithm is mainly determined by the computation of the stepsize for solving the subproblem of $\mathbf{X}$, where $2 N^{2} M / L$ flops are needed, which is less than the symbol-level methods. Moreover, as the RS factors only need to be communicated to the users once per $L$ symbols, the incurred signaling overhead would be considerably reduced.

\section{NUMERICAL RESULTS}

In this section, we provide numerical results to evaluate the performance of our proposed CE scheme. The following four algorithms are considered:

- The conventional alternating optimization method (AO).

- The simplified AO algorithm (Simplified AO).

- The frame-level AO algorithm (Frame-level AO).

- The RCG algorithm based on CI (CI-RCG) [8].

Without loss of generality, we set $P_{T}=1 . N=64$ and $\Delta=10^{-6}$ (the maximum torerance) for all the proposed algorithms. The maximum number of iterations we set for $r_{k}$ is 1000. For frame-level algorithm, we set $L=8$ (the number of symbols) i.e. an RS factor is sent to a user per 16 bits, assuming QPSK modulation. Fig.1(a) shows the SER performance of algorithms with increased transmitted signalto-noise ratio (SNR), where $\mathrm{SNR}=P_{T} / \sigma^{2}$. It is worth noting 


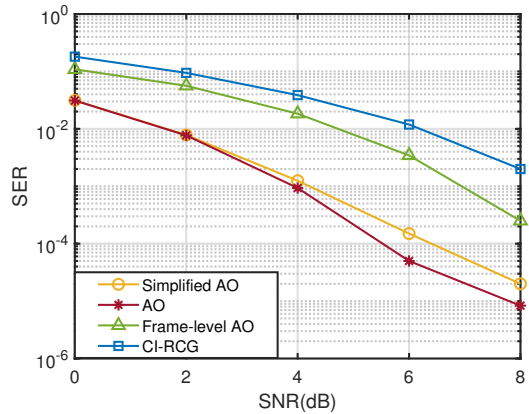

(a) SER versus SNR for different algorithms, $M=20$.

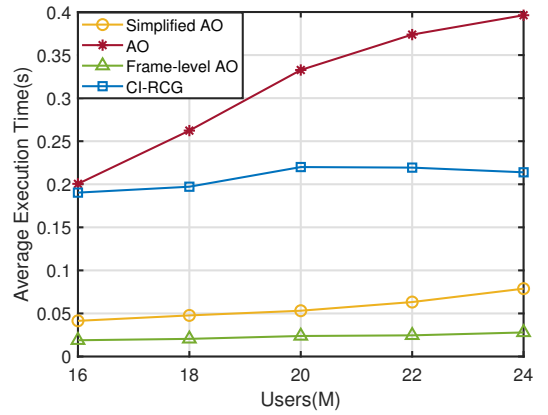

(b) Average execution time versus number of users for different algorithms.

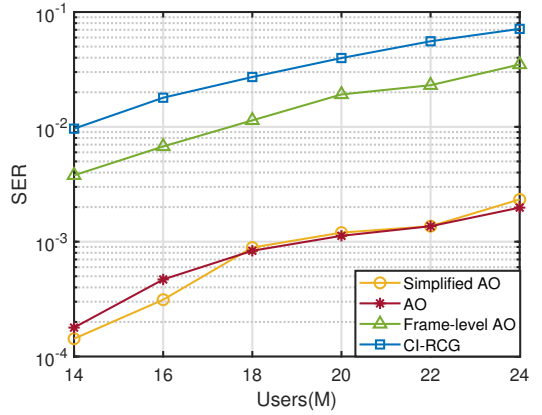

(c) SER versus user for different algorithms, $S N R=$ $4 d B$.

Fig. 1: Numerical results.

that the conventional $\mathrm{AO}$ method and its simplified counterpart have similar SER performance, both of which significantly outperform the CI algorithm for CE. And the frame-level method is also superior to the latter.

In order to verify the advantages of our algorithms in terms of the complexity, we compared the average execution time of the four algorithms with different number of users with a fixed BS antenna number, and a fixed SNR. The results are shown in Fig.1(b). While the conventional and simplified AO algorithms have similar SER performance, the computational complexity of the conventional method is much higher. Moreover, we observe that the RCG-CI is more efficient than the frame-level design, at the price of significantly higher SER. The results show that our proposed algorithms can simultaneously achieve complexity reduction and SER performance improvements compared to the state-of-the-art CEP design [8].

In Fig.1(c), we further look at the SER performance with increasing users, where the number of users ranges from 14 to 24 , and the SNR is fixed at $4 \mathrm{~dB}$. Again, we see that our algorithm achieves lower SER than that of RCG.

\section{CONCLUSION}

This letter presents a novel CEP design for the scenario of the downlink MU-MISO. In particular, we minimize the summation of the inverse SINR of all the users by carefully designing both the transmit signal matrix and the RS factors for each user. The proposed algorithm optimizes variables alternatively based on gradient projection and Dinkelbach methods. For the purpose of reducing the complexity and signaling overheads, we further propose a simplified $\mathrm{AO}$ algorithm and a frame-level RS factor design. The simulation results show that our proposed methods achieve a superior performance in terms of both computational complexity and SER.

\section{REFERENCES}

[1] S. K. Mohammed and E. G. Larsson, "Per-antenna constant envelope precoding for large multi-user mimo systems," IEEE Transactions on Communications, vol. 61, no. 3, pp. 1059-1071, 2013.

[2] J. Chen, C. Wen, and K. Wong, "Improved constant envelope multiuser precoding for massive mimo systems," IEEE Communications Letters, vol. 18, no. 8, pp. 1311-1314, 2014.

[3] J. Pan and W. K. Ma, "Constant envelope precoding for single-user largescale miso channels: Efficient precoding and optimal designs," IEEE Journal of Selected Topics in Signal Processing, vol. 8, no. 5, pp. 982995, 2014.

[4] S. Zhang, R. Zhang, and T. J. Lim, "Constant envelope precoding with adaptive receiver constellation in miso fading channel," IEEE Transactions on Wireless Communications, 2016.

[5] S. Zhang, R. Zhang, and T. J. Lim, "Miso multicasting with constant envelope precoding," IEEE Wireless Communications Letters, vol. 5, no. 6, pp. 588-591, 2016.

[6] S. Zhang, R. Zhang, and T. J. Lim, "Constant envelope precoding for mimo systems," IEEE Transactions on Communications, vol. 66, no. 1, pp. 149-162, 2018.

[7] P. V. Amadori and C. Masouros, "Constant envelope precoding by interference exploitation in phase shift keying-modulated multiuser transmission," IEEE Transactions on Wireless Communications, vol. 16, no. 1, pp. 538-550, 2017.

[8] F. Liu, C. Masouros, P. V. Amadori, and H. Sun, "An efficient manifold algorithm for constructive interference based constant envelope precoding," IEEE Signal Processing Letters, vol. 24, no. 10, pp. 1542-1546, 2019.

[9] J. Tranter, N. D. Sidiropoulos, X. Fu, and A. Swami, "Fast unit-modulus least squares with applications in beamforming," IEEE Transactions on Signal Processing, vol. 65, no. 11, pp. 2875-2887, 2017.

[10] O. Castañeda, T. Goldstein, and C. Studer, "Pokemon: A non-linear beamforming algorithm for 1-bit massive mimo," in 2017 IEEE International Conference on Acoustics, Speech and Signal Processing (ICASSP), 2017, pp. 3464-3468.

[11] D. W. K. Ng, E. S. Lo, and R. Schober, "Wireless information and power transfer: Energy efficiency optimization in ofdma systems," IEEE Transactions on Wireless Communications, vol. 12, no. 12, pp. 6352$6370,2013$.

[12] Z. Xu, H. Zhang, Y. Xu, and G. Lan, "A unified single-loop alternating gradient projection algorithm for nonconvex-concave and convexnonconcave minimax problems," 2020. 